\title{
Role of endogenous cholecystokinin on growth of human pancreatic cancer
}

\author{
GAIL L. MATTERS ${ }^{1}$, CHRISTOPHER McGOVERN ${ }^{2}$, JOHN F. HARMS $^{3}$, KEVIN MARKOVIC $^{3}$, KRYSTAL ANSON $^{2}$, \\ CALPURNIA JAYAKUMAR ${ }^{2}$, MELISSA MARTENIS ${ }^{2}$, CHRISTINA AWAD ${ }^{3}$ and JILL P. SMITH ${ }^{2}$ \\ Departments of ${ }^{1}$ Biochemistry and Molecular Biology, and ${ }^{2}$ Department of Medicine \\ The Pennsylvania State University College of Medicine, 500 University Drive, Hershey, PA 17033; \\ ${ }^{3}$ Department of Biological Sciences, Messiah College, Grantham, PA 17027, USA
}

Received August 11,2010; Accepted September 13, 2010

DOI: $10.3892 /$ ijo. 2010.886

\begin{abstract}
Cholecystokinin (CCK) and gastrin stimulate growth of pancreatic cancer. Although down-regulation of gastrin inhibits growth of pancreatic cancer, the contribution of endogenous CCK to tumor growth is unknown. The purpose of this study was to evaluate the role of endogenous CCK on autocrine growth of pancreatic cancer. Pancreatic cancer cell lines were analyzed for CCK mRNA and peptide expression by real-time RT-PCR and radioimmunoassay, respectively. The effect of endogenous CCK on growth was evaluated by treating cancer cells with CCK neutralizing antibodies and by down-regulating CCK mRNA by RNAi. Wild-type pancreatic cancer cells expressed significantly lower CCK mRNA and peptide levels than gastrin. Neither treatment of pancreatic cancer cells with CCK antibodies nor the down-regulation of CCK mRNA and peptide by shRNAs altered growth in vitro or in vivo. Conversely, when gastrin mRNA expression was down-regulated, the same cells failed to produce tumors in spite of having sustained levels of endogenous CCK. Pancreatic cancer cells produce CCK and gastrin; however, the autocrine production of gastrin is more important for stimulating tumor growth.
\end{abstract}

\section{Introduction}

Growth factors which regulate cancer cell function have become prime targets for cancer therapy, including pancreatic cancer. One newer method for targeting cancers has included novel therapeutics based on RNA interference (RNAi) toward growth factors; (1) however, validated RNAi targets must first be designed and tested in vitro and in animal

Correspondence to: Professor Jill P. Smith, Division of GI and Hepatology, Penn State College of Medicine, 500 University Drive, Hershey, PA 17011, USA

E-mail: jsmith2@psu.edu

Key words: gastrin, RNAi, pancreatic cancer, tumor growth, immunocytochemistry, cholecystokinin models. The gastrointestinal peptides CCK and gastrin are potential targets for cancer therapeutics since both of these peptides have been shown to stimulate growth of this malignancy $(2,3)$ through the $\mathrm{CCK}_{2}$ (or CCK-B) receptor with gastrin having a greater affinity than $\mathrm{CCK}$ for this receptor in malignant cells (4-6).

The role of gastrin in autocrine growth regulation of pancreatic cancer has been established (7). Normal human and porcine pancreas contain small amounts of bioactive amidated gastrins in the fetal pancreas $(8,9)$ however, after birth, gastrin immunoreactivity is found primarily located in the G-cells of the gastric antrum and not in the adult pancreas (10). In contrast, gastrin immunoreactivity has been identified in human pancreatic cancer cells and tissues $(3,7,11)$ suggesting the re-expression or an over-expression of this peptide in the malignant state and a role in the development of this cancer (12). The fully processed form of gastrin, gastrin-17, was detected in cultured pancreatic cancer cells and their growth medium indicating that this peptide was secreted from the cells and in surgically removed pancreatic cancer specimens from patients by immunohistochemistry and radioimmunoassay (RIA) $(7,9,13)$. Down-regulation of gastrin gene expression by RNAi decreases tumor gastrin peptide production, tumor growth and metastases (14).

Although exogenous administration of CCK to pancreatic cancer cells also stimulates growth $(2,15)$ the role of CCK as an autocrine growth regulator in pancreatic cancer has not been examined. Goetze and colleagues (16) assessed human pancreatic cancer surgical specimens for gastrin and CCK peptide and mRNA expression by radioimmunoassay and RT-PCR, respectively. Although this group found high levels of $\alpha$-amidated gastrins and its precursor forms in $74 \%$ of the pancreatic tumor specimens, CCK was not found (16). In contrast, Tamiolakis and coworkers (17) reported CCK immunoreactivity in 10 out of 15 surgical specimens with the CCK-positive cells identified in the duct-like structures of the pancreatic tumors. Therefore, the literature has conflicting reports on whether pancreatic cancer cells synthesize CCK and whether this peptide serves a physiological role in the stimulation of cancer cell growth.

In the current investigation, we evaluated the quantitative expression and function of autocrine CCK in human pancreatic 
cancer cells. CCK mRNA levels in pancreatic cancer cells were measured by real-time RT-PCR and CCK peptide levels were quantified by RIA. By using RNA interference and antisense technologies that specifically down-regulate either CCK or gastrin mRNA expression, the role of these peptides on cancer cell growth was studied.

\section{Materials and methods}

Cell lines. The human pancreatic cancer cell lines used in these experiments were purchased from the ATCC (Rockville, $\mathrm{MD}$ ), and were maintained in the appropriate media: DMEM with $10 \%$ FBS for PANC-1 and MIA PaCa-2, Iscove's with $20 \%$ FBS for Capan-1 and RPMI-1640 with 10\% FBS for BxPC-3 and AsPC-1.

Measurement of CCK, gastrin, $C C K_{1}$ receptor and $\mathrm{CCK}_{2}$ receptor $m R N A$ by $R T$-PCR in pancreatic cancer cells. RNA was extracted from wild-type human ductal pancreatic cancer cells using RNeasy kits (Qiagen, Valencia, CA). For real-time RT-PCR, RNA was quantified on an RNA 6000 Nanochip using the Agilent Bioanalyzer 2100. First-strand cDNA was produced from $1.0 \mu \mathrm{g}$ of total RNA using random hexamer primers and a SuperScript III reverse transcription kit (Invitrogen, Carlsbad, CA). cDNA (100 ng per reaction) was analyzed using TaqMan Gene expression assays for CCK (assay ID Hs00174937_m1), gastrin (assay ID Hs00174945_m1), CCK receptor (assay ID Hs00167891_m1), and $\mathrm{CCK}_{2}$ receptor (assay ID Hs00176123_m1) with cyclophilin A (assay ID Hs99999904_m1) as the internal control (Applied Biosystems, Foster City, CA). To exclude the possibility of genomic DNA contamination, control reactions with no cDNA template were performed. PCR amplification and analysis were done with the Applied Biosystems Sequence Detection System 7700 using the relative quantification $(\mathrm{ddCt})$ plate setup. RNA from wild-type PANC-1 cells was used as an expression calibrator for each target.

Detection of CCK peptide by immunocytochemistry in pancreatic cancer cells. BxPC-3 cells were plated onto $22-\mathrm{mm}^{2}$ round glass coverslips and grown in complete media for $36-48 \mathrm{~h}$. After the media were removed, the cells were washed and fixed in $2 \%$ formaldehyde for $15 \mathrm{~min}$ at $4^{\circ} \mathrm{C}$. Cells were rinsed twice with $1 \mathrm{X}$ phosphate-buffered saline (PBS), permeabilized with $0.25 \%$ Triton $\mathrm{X}-100$ for $10 \mathrm{~min}$ followed by incubation in $100 \mathrm{mM}$ glycine in PBS for $5 \mathrm{~min}$ at room temperature and an additional $1 \mathrm{X}$ PBS rinse. Nonspecific antibody binding was blocked with $5 \%$ normal goat serum in PBS for $1 \mathrm{~h}$ at room temperature. Cells were incubated in the same buffer containing a rabbit polyclonal anti-CCK antibody (C2581, Sigma, St. Louis, MO) at a titer of 1:5,000 overnight at $4^{\circ} \mathrm{C}$. After 3 washes in PBS, the cells were incubated in PBS containing $2 \%$ normal goat serum and a goat anti-rabbit Alexa Fluor 568-labeled secondary antibody (Invitrogen) $(1: 2,000)$ for $2 \mathrm{~h}$ at room temperature. The slides were washed 2 times with PBS for 30 min each and mounted on slides using DAPI medium for visualization of nuclei. The negative control was wild-type BxPC-3 cells incubated with secondary antibody alone $(1: 2,000)$. Immunofluorescent staining was visualized and photographed using a Leica TCS
SP2 AOBS confocal microscope using $63 \mathrm{X}$ objective at the appropriate laser settings.

In vitro growth studies with selective peptides and anti-CCK antibodies. CCK-8 (sulfated, MW 1143.29) and gastrin-17 (sulfated, MW 2178.30) were purchased from Peninsula Laboratories (San Carlos, CA). Pancreatic cancer cells were adapted to grow in serum-free media, and growth rates were then evaluated in the presence of exogenously administered gastrin or CCK. Cells $\left(1 \times 10^{5}\right)$ were plated in each well of a 12-well plate and treated with various concentrations $\left(10^{-11}-10^{-7} \mathrm{M}\right)$ of gastrin or CCK (6 wells for each concentration). Fresh media with or without the indicated peptide was added daily and viable cells were counted by hemacytometer after 6 days of treatment.

A series of experiments were designed to examine the effect of decreasing the levels of CCK peptide secreted from pancreatic cancer cells into growth media. Two different CCK antibodies against either fully processed CCK or proCCK were used. The first antibody, Ab89009, was a polyclonal rabbit antibody raised against the non-processed $\mathrm{N}$-terminus sequence (62-71) of human proCCK and was a gift of Professor Jens Rehfeld (18). This antibody has no detectable cross-reactivity with gastrin or fully processed CCK-8 (18). The second polyclonal antibody, C2581, was purchased from Sigma-Aldrich, and was produced using as an immunogen the synthetic-sulfated cholecystokinin (26-33) amide (sulfated CCK-8), conjugated to KLH. Low cross reactivity is observed with gastrin-17 (0.2\%) and gastrin-34 (1.0\%). Both of these CCK antibodies functionally neutralize the capacity of CCK to bind to CCK receptors.

CCK antibody experiments were performed with both BxPC-3 cells (which express moderate-CCK mRNA levels and high-gastrin mRNA levels) and PANC-1 cells (which express high-CCK mRNA and low-gastrin mRNA levels). BxPC-3 cells or PANC-1 $\left(4 \times 10^{4}\right)$ were plated in 12-well tissue culture dishes and grown in serum-free media on day 0 . Cells were treated in different experiments with either the proCCK antibody Ab89009 (BxPC-3) or the fully-processed antibody CCK AbC2581 (BxPC-3 and PANC-1) in dilutions ranging from 1:5,000-1:500. Control cells were treated with either a non-immune IgG (Sigma) as a non-specific antibody control, or media only. Cells were treated at two-time intervals: the first at $24 \mathrm{~h}$ after plating and the second at $96 \mathrm{~h}$ after plating. After 7 days of growth, cells were harvested and viable cells counted using the trypan blue exclusion technique.

Down-regulation of CCK with RNAi and antisense transfection. BxPC-3 cells were independently transfected with one of two shRNA (short hairpin RNA) constructs targeting independent, non-overlapping regions of human CCK mRNA. shRNA site selection was based upon open mRNA secondary structure and low off-target effects. The shRNA target sequences used are shown in Table I and started at nucleotide positions -6 and +141 relative to the CCK mRNA translation start site. The gastrin shRNA target site sequence started at nucleoside position +286 on the gastrin mRNA. BLAST analysis revealed no significant homologies between the sequences and mRNAs other than human CCK or gastrin. Non-specific shRNA control plasmids containing an shRNA 
Table I. CCK and gastrin shRNA target sequences.

\begin{tabular}{cc}
\hline Target site & \multicolumn{1}{c}{ Sequence } \\
\hline -6 CCK mRNA & 5'-AAAGCCAUGAACAGCGGC-3' \\
+141 CCK mRNA & 5'-CAGAGAACGGAUGGCGAGU-3' \\
+286 gastrin mRNA & 5'-GUGCUGAGGAUGAGAACUA-3' \\
\hline
\end{tabular}

oligonucleotide duplex with no homology to any mammalian gene sequence (Oligoengine, Seattle, WA) served as a control for general cellular responses to double-stranded RNA. Annealed oligonucleotide duplexes were cloned into the pSUPER vector (Oligoengine) containing a hygromycin resistance gene for $\mathrm{CCK}$ clones and neomycin resistance gene for gastrin clones for selection. Plasmid constructs were transfected into BxPC-3 pancreatic cancer cells with Lipofectamine 2000 (Invitrogen). At least 50 stable BxPC-3 clonal transfectants were selected for each of the shRNA constructs. CCK and gastrin mRNA knockdown was assessed by real-time RT-PCR. The generation of the BxPC-3 gastrin knockdown clone sh286, which is also used in this study, has been previously published (14).

Additionally, a full-length human CCK antisense cDNA in vector pcDNA3.1(-) (Invitrogen) was transfected into BxPC-3 pancreatic cancer cells using Lipofectamine 2000. Control cells were treated with empty vector DNA. Stable clones were selected and CCK mRNA knockdown was confirmed by RT-PCR.

Measurement of CCK peptide in human pancreatic cancer cells and clones by radioimmunoassay. BxPC-3 pancreatic cancer cells and clones that had been stably transfected with either non-specific shRNA, CCK shRNA (141 or -6), antisense CCK DNA, or empty vector were grown in cell culture to log-phase at which time the media were removed and replaced with fresh media. After $24 \mathrm{~h}$ of further growth, the cells were rinsed, scraped, and cellular extracts collected in $\mathrm{dH}_{2} \mathrm{O}$ containing $1 \mathrm{X}$ Complete Mini protease inhibitor (Roche, Indianapolis, IN). CCK peptide radioimmunoassay was performed in duplicate using a RIA kit from ALPCO Diagnostics (Windham, NH) with rabbit antiserum to synthetic CCK 26-33, sulfate and ${ }^{125} \mathrm{I}-\mathrm{BH}$ CCK-8. This antibody has $0.5 \%$ cross reactivity to sulfated gastrin- 17 and $<0.01 \%$ cross reactivity to non-sulfated gastrin-17. Samples were standardized by protein concentration using a Micro BCA protein assay with albumin as standard (Pierce, Rockford, IL).

In vivo tumor growth. Athymic nude mice (Harlan, 6-week male $n u / n u$ ) were used for in vivo tumor growth experiments. The Institutional Animal Care and Usage Committee of the Pennsylvania State University College of Medicine approved the research protocol and animals were housed in accordance with the AAALAC guidelines for veterinary medicine. BxPC-3 cells were harvested from culture flasks with trypsin, centrifuged and washed. Cells $\left(1 \times 10^{6}\right)$ were suspended in $0.1 \mathrm{ml}$ of Hank's buffered saline solution (HBSS) and injected subcutaneously into the right and left flanks of each mouse. Tumor diameter was measured weekly with calipers and tumors were removed if the mean tumor diameter exceeded $12 \mathrm{~mm}$. At least 6 animals were analyzed for each of the following clones ( $\mathrm{N}=12$ tumors per group): CCK shRNA $-6, \mathrm{CCK}$ shRNA +141, non-specific shRNA control (NSC), or gastrin shRNA 286.

Statistical analysis. Real-time RT-PCR groups were compared by ANOVA and pair wise t-tests with a Bonferroni correction for multiple comparisons. Real-time RT-PCR results were expressed as a relative quantity according to the method of Livak and Schmittgen (19). In vivo tumor growth was analyzed using pair wise Mann-Whitney tests on groups as indicated. The data from each clone were pooled and analyzed using mean values. Significance of two-sided statistical tests was considered to be $\mathrm{p}<0.05$.

\section{Results}

Quantification of CCK MRNA in human pancreatic adenocarcinoma cell lines. Pancreatic cancer cells were analyzed for their endogenous expression of CCK peptide, gastrin peptide, $\mathrm{CCK}_{1}$ receptor and $\mathrm{CCK}_{2}$ receptor mRNA by real-time RT-PCR. CCK mRNA was detected in 4 of 5 human pancreatic adenocarcinoma cell lines analyzed. Quantification of CCK mRNA levels by real-time RT-PCR revealed that Capan-1 had high CCK mRNA levels (Fig. 1A). PANC-1, BxPC-3 and AsPC-1 expressed intermediate levels of CCK mRNA and CCK mRNA from MIA PaCa-2 cells was undetected by real-time RT-PCR; therefore MIA PaCa- 2 cells were excluded from the pool of potential cancer cells to examine autocrine effects of CCK peptide.

Gastrin mRNA expression from the same cell lines is shown in Fig. 1B revealing gastrin mRNA was detected at levels that exceeded that of CCK peptide mRNA in all cell lines except PANC-1 cell which had comparable levels. CCK $_{1}$ receptor mRNA (Fig. 1C) was detected in PANC-1 and Capan-1 cancer cells. All cancer cell lines examined had detectable $\mathrm{CCK}_{2}$ receptor mRNA (Fig. 1D).

The presence and localization of intracellular CCK peptide was examined by immunocytochemistry in wild-type BxPC-3 cancer cells (Fig. 1E). CCK immunoreactivity was found predominantly in the cytoplasm but slight nuclear immunoreactivity was also observed. This pattern of staining previously described by Tamiolakis and colleagues (17) appears to be characteristic of CCK immunoreactivity.

Gastrin is a more potent stimulant of pancreatic cancer cell proliferation. In BxPC-3 cancer cells, exogenous gastrin application stimulated cell growth with concentrations of gastrin that were 100-fold lower than the concentration of $\mathrm{CCK}$ and with a more profound response (Fig. 2A). BxPC-3 cells grown in serum-free media alone (controls) underwent 3.5 doublings over a 6-day time period, while cells treated with $10^{-9} \mathrm{M}$ gastrin increased $68 \%$ over control cells (Fig. 2A). In contrast, cells treated with CCK- 8 only increased by $36 \%$ over controls when treated with a concentration of CCK that was 100 -fold greater $\left(10^{-7} \mathrm{M}\right)$ than the gastrin concentration which stimulated growth (Fig. 2B).

Decreasing CCK peptide levels with neutralizing CCK antibodies has no effect on in vitro growth. To test the effect 
A

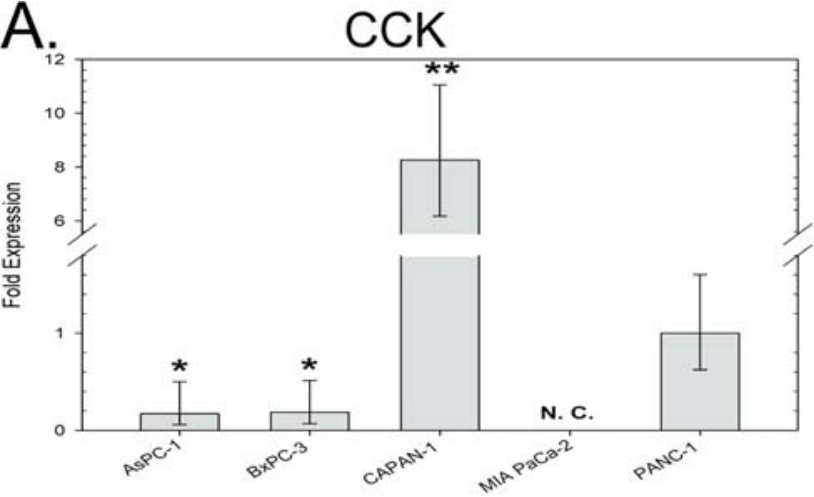

C
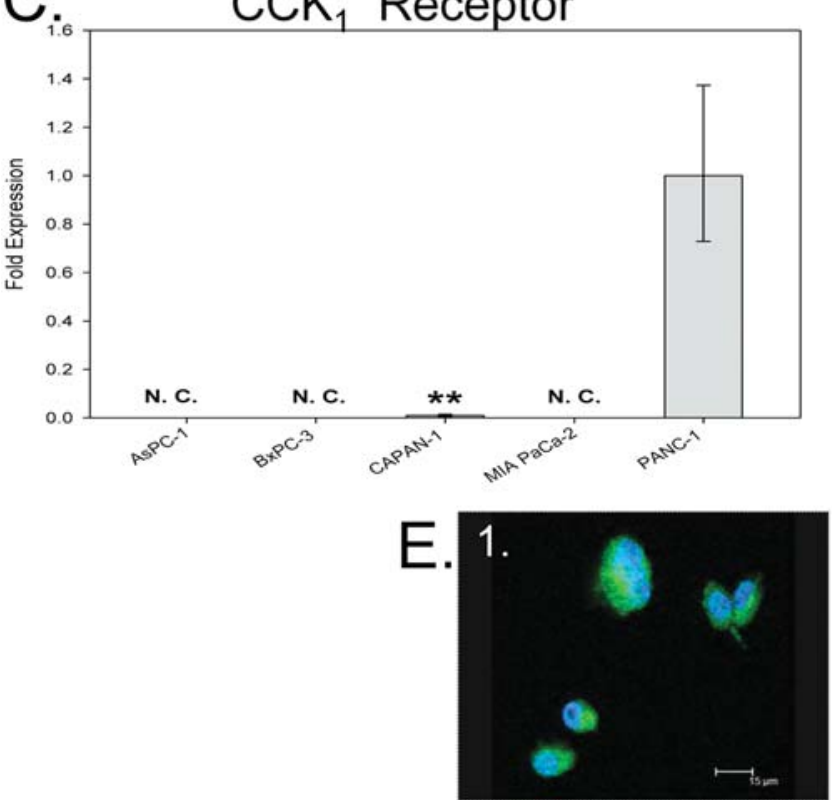

B.
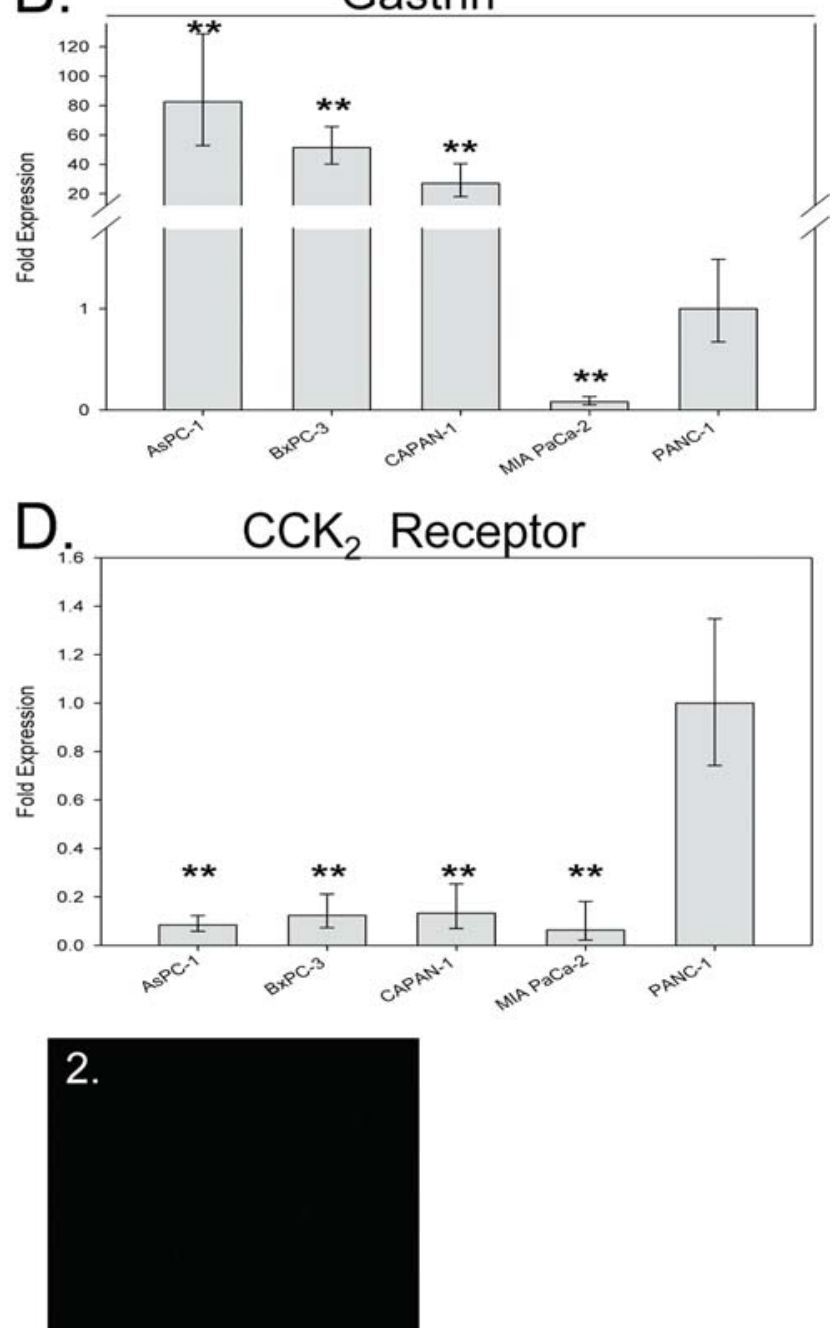

Figure 1. Quantification of CCK, gastrin, $\mathrm{CCK}_{1}$ receptor and $\mathrm{CCK}_{2}$ receptor $\mathrm{mRNA}$ expression in human pancreatic cancer cell lines by real-time RT-PCR. Human cultured pancreatic cancer cell lines AsPC-1, BxPC-3, Capan-1, MIA PaCa2 and PANC-1 were analyzed for the expression of CCK mRNA (A), gastrin mRNA (B), $\mathrm{CCK}_{1}$ receptor mRNA (C) and $\mathrm{CCK}_{2}$ receptor mRNA (D) by real-time RT-PCR. The presence of CCK peptide in BxPC-3 cells was verified by immunocytochemical staining (E1) with an anti-CCK antibody. Cells were reacted with an anti-human CCK antibody followed by a goat antirabbit Alexa Fluor 568 labeled secondary antibody. Negative control cells (E2) were stained with secondary antibody alone. Asterisks $\left({ }^{*} \mathrm{p}<0.05,{ }^{* *} \mathrm{p}<0.001\right)$ indicate values significantly different from that in PANC-1 cells, which express all four gene products. N.C., not called.

A.

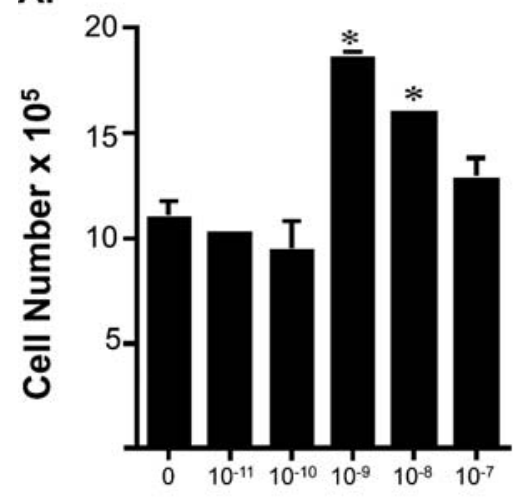

Gastrin $17(\mathrm{M})$
B.

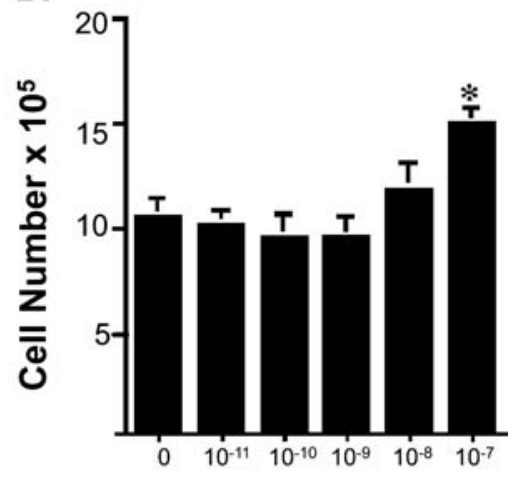

CCK 8 (M)

Figure 2. Comparison of effects of exogenous gastrin or CCK on growth of BxPC-3 cells in vitro. Cells were grown in serum-free medium in the presence of either CCK or gastrin $\left(10^{-11}-10^{-7} \mathrm{M}\right)$ for 6 days, with fresh media with or without the indicated peptide added daily. Gastrin stimulated cancer cell growth twice that of CCK at a 100-fold lower concentration of gastrin. Bars represent the standard error of the mean of 12 replicates. Significantly different from controls ${ }^{*} \mathrm{p}<0.05$. 
A.

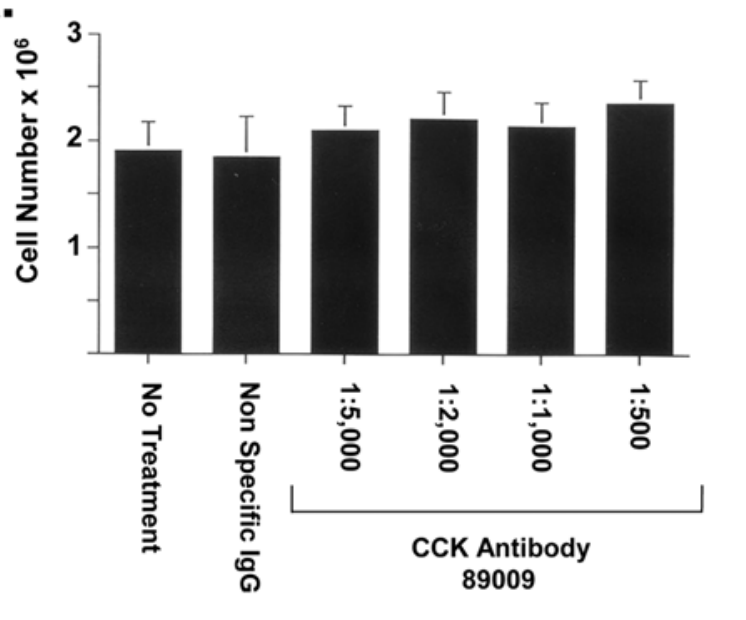

B.

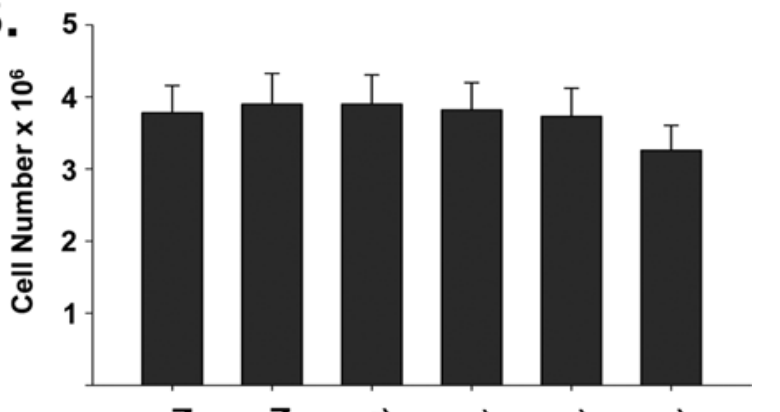

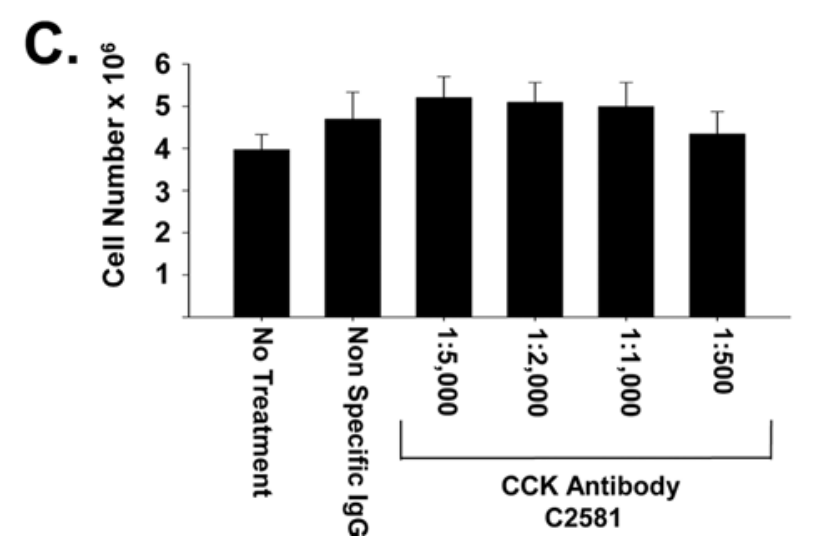

Figure 3. Effects of selective, neutralizing CCK antibodies on the growth of pancreatic cancer cells in vitro. Treatment of BxPC-3 cells with increasing amounts of antibody against proCCK (A) or fully processed CCK (B) over the course of one week did not inhibit cell proliferation. PANC-1 cells treated with varying concentrations of anti-CCK antibody (C2581) in serum-free media also grew at the same rate as untreated cells or as cells treated with a non-immune $\operatorname{IgG}(\mathrm{C})$. Bars represent the standard error of the mean of 12 replicates. Significantly different from controls * $\mathrm{p}<0.05$.

of reducing levels of endogenously produced CCK peptide on in vitro growth of wild-type BxPC-3 cells, two neutralizing CCK antibodies were used (Ab89009 and C2581). Neither CCK antibody at dilutions of up to 1:500 had any effect cell growth over a one-week period (Fig. 3A and B). There was no effect on cell number when another pancreatic cancer cell line, PANC-1 cancer cells, was treated with the CCK antibody, C2581 (1:500) compared to media alone or nonimmune IgG (Fig. 3C).

Down-regulation of CCK using RNAi. BxPC-3 was chosen as the recipient cell line for $\mathrm{CCK}$ peptide down-regulation for several reasons. First, this cell line has an intermediate level of expression of both CCK and gastrin mRNA and expresses only the $\mathrm{CCK}_{2}$ receptor (not the $\mathrm{CCK}_{1}$ receptor), which binds both peptides equally. Second, it is known that the addition of either gastrin (3) or CCK (2) peptide to culture media stimulates BxPC-3 growth in vitro, indicating that this cell line has a physiologically relevant response to both of these peptides. Third, previous work with BxPC-3 cells showed that this cell line contains levels of gastrin that are comparable to those found in fresh human surgical pancreatic tumors, making it a representative model for study (13). Thus, we chose to use this cell line in order to make a direct comparison of the growth effects of CCK knockdown to the growth effects of gastrin knockdown.

Although $\mathrm{CCK}_{1}(\mathrm{CCK}-\mathrm{A})$ receptors have previously been described in pancreatic cancer tissues (20), the role and significance of these receptors is unknown since CCKstimulated growth in pancreatic cancer is mediated through the $\mathrm{CCK}_{2}$ receptor (5). Since CCK peptide binds to the $\mathrm{CCK}_{1}$ receptor with greater affinity (21) than the $\mathrm{CCK}_{2}$ receptor, we specifically selected a cell line that lacked $\mathrm{CCK}_{1}$ receptors to target only the $\mathrm{CCK}_{2}$ receptor which is involved in cell proliferation. Although PANC-1 and Capan-1 make more CCK mRNA than BxPC-3, these cell lines were not chosen for the down-regulation experiments for several additional reasons. First, the effects of CCK or gastrin on growth of Capan-1 in vitro or in vivo have not been described. Second, PANC-1 and Capan-1 cells both express the $\mathrm{CCK}_{1}$ receptor (Fig. 1); therefore, neither of these two cell lines were selected for the transfection experiments to eliminate the offtarget effect. Use of selective $\mathrm{CCK}_{1}$ and $\mathrm{CCK}_{2}$ receptor antagonists has demonstrated that the growth stimulation by CCK (22) and gastrin (3) is mediated through the $\mathrm{CCK}_{2}$ receptor and that blockade of the $\mathrm{CCK}_{1}$ receptor does not influence the growth rate of pancreatic cancer cells. Third, 
A.

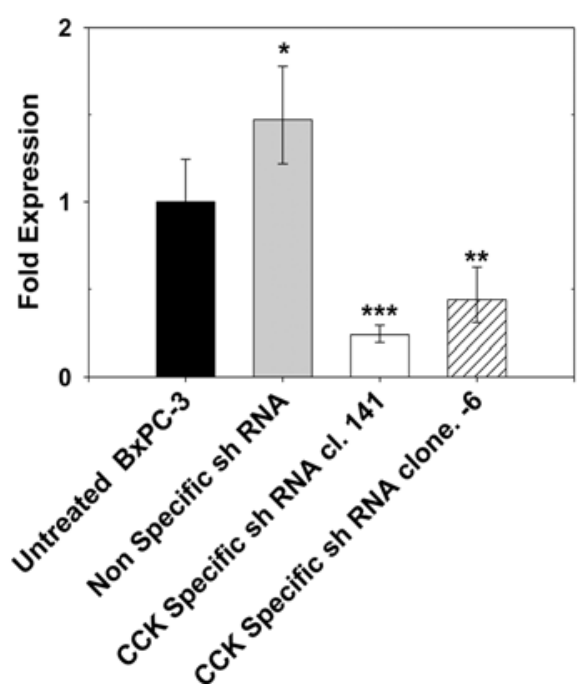

B.

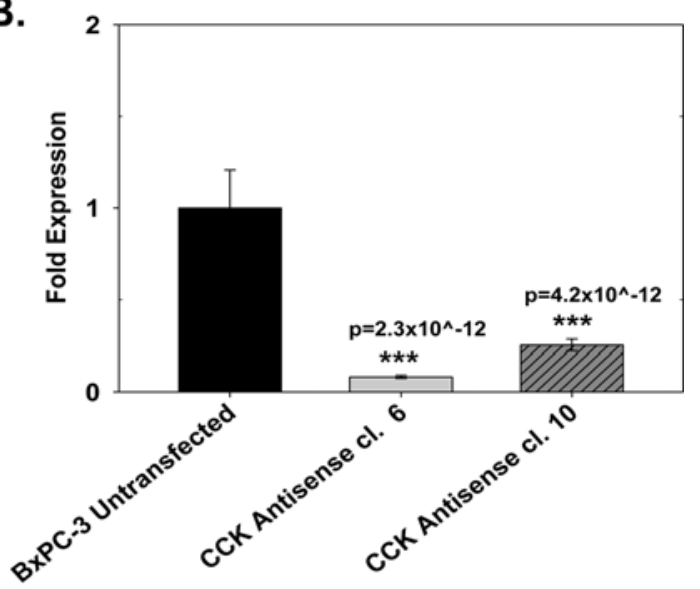

Figure 4. CCK mRNA levels in pancreatic cancer CCK down-regulated clones and controls. (A) Real-time RT-PCR analysis of BxPC-3 cells shows significantly less CCK mRNA in clones stably transfected with either of two different CCK shRNA constructs (shRNA -6 or shRNA +141) compared to a nonspecific control shRNA (NSC) or wild-type cells. Columns represent the fold change in mRNA levels calculated from the mean relative quantity (RQ $=2^{-\Delta \Lambda C T}$ ) and bars represent a 95\% confidence interval $\left(C I ; R Q=2^{-(\Delta \Lambda C T \pm C I}\right)$. (B) Real-time RT-PCR for CCK mRNA in CCK antisense-transfected clones. Antisense clones 6 and 10 had decreased CCK mRNA expression. Significantly different from untransfected control cells at ${ }^{*} \mathrm{p}<0.05,{ }^{* *} \mathrm{p}<0.001$, or ${ }^{* * *} \mathrm{p}<0.0001$ or specific p-values as noted.

the impact of gastrin knockdown on Capan-1 tumor growth has not been assessed, so a direct comparison of the effects of gastrin and CCK knockdown on tumor growth cannot be made. For all these reasons, BxPC-3 cancer cells were selected for the RNAi experiments.

Cholecystokinin mRNA expression was effectively decreased with both antisense and shRNA transfection (Fig. 4). Cells transfected with either the CCK shRNA -6 or the shRNA +141 construct showed significant reduction in CCK mRNA levels compared to non-specific (NSC)-transfected control clones as assessed by real-time RT-PCR was performed. Compared to untransfected cells, CCK mRNA levels in the CCK shRNA -6 and shRNA +141 clones were significantly decreased by $56 \%\left({ }^{* *} \mathrm{p}<0.001\right)$ and $76 \%\left({ }^{* * *} \mathrm{p}<0.0001\right)$, respectively (Fig. 4A). The non-specific shRNA clones had slightly more CCK mRNA than untransfected, wild-type cells thought to be due to clonal variation. Antisense downregulation of CCK mRNA was found in two BxPC-3 clones (6 and 10) (Fig. 4B). These two antisense clones were selected for further analysis of CCK peptide levels by RIA.

CCK peptide levels in pancreatic cancer cells and CCK knockdown clones by radioimmunoassay. CCK peptide as measured by RIA in cellular extracts was decreased by $60 \%$ in CCK shRNA -6 clones compared to wild-type cells, whereas the CCK shRNA +141 clones did not show a significant decrease in CCK peptide (Table II). Both clones transfected with the CCK antisense cDNA showed decreased CCK peptide compared to controls, but the decreased peptide expression was not as pronounced as that obtained with the CCK RNAi using shRNA -6 .

Influence of CCK and gastrin on tumor growth in vivo. BxPC-3 clones with reduced CCK mRNA levels were tested
Table II. CCK peptide radioimmunoassay.

\begin{tabular}{lc}
\hline $\begin{array}{l}\text { Cellular extracts of } \\
\text { BxPC-3 cells and clones }\end{array}$ & $\begin{array}{c}\text { CCK } \\
\text { (pg/mg protein) }\end{array}$ \\
\hline Wild-type & 1.25 \\
NSC shRNA (control) & 1.07 \\
Empty vector antisense (control) & 1.36 \\
CCK shRNA +141 & 1.64 \\
CCK shRNA -6 & $0.50^{\mathrm{a}}$ \\
CCK antisense clone 10 & 0.91 \\
CCK antisense clone 6 & $0.71^{\mathrm{a}}$
\end{tabular}

${ }^{a} \mathrm{p}<0.05$ indicates values significantly different from that in wildtype BxPC- 3 cells.

for their ability to form tumors in vivo. The rate of tumor growth corresponded to the amount of CCK peptide detected in the same cells by RIA (Fig. 5). The CCK shRNA +141 clone grew fastest and also had the greatest amount of CCK peptide detected by RIA. The CCK shRNA - 6 cells grew the slowest of the CCK-down-regulated clones in animals and this clone had the least amount of CCK peptide detected by RIA. Although CCK mRNA levels were reduced in up to $76 \%$ in the CCK shRNA +141 clones, it appears that tumor growth is more dependent upon post-transcriptional processing, i.e., peptide levels. However, neither of the CCK-downregulated clones grew statistically different from the controls. BxPC-3 cells with decreased gastrin expression developed significantly smaller tumors over the 5-week period of time (Fig. 5). The relationship between tumor growth at 5 weeks 


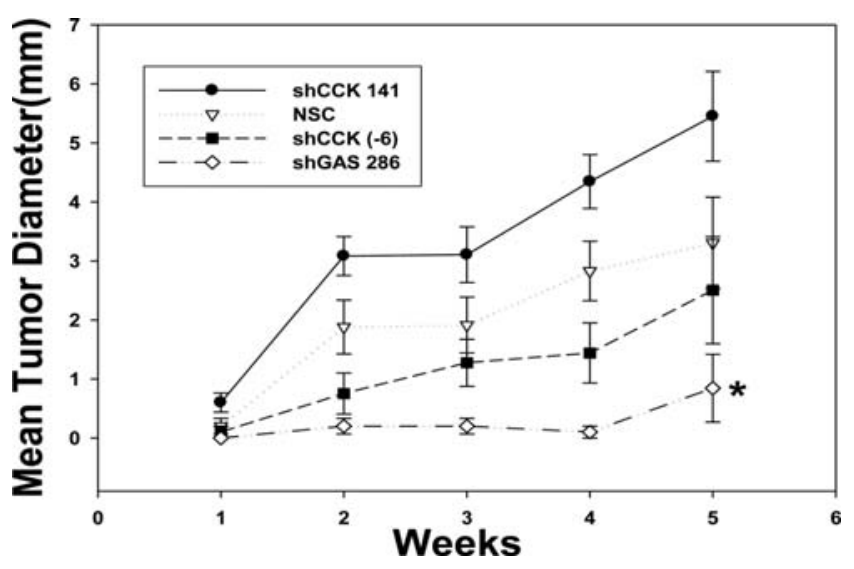

Figure 5. Down-regulation of gastrin but not CCK decreases tumor growth of pancreatic cancer in vivo. Growth of BxPC-3 CCK knock-down clones was measured as subcutaneous tumors in nude mice over five weeks. There were no significant differences in tumor growth of BxPC-3 cells in which CCK peptide was down-regulated compared to a non-specific control clone In contrast, tumors from a $\mathrm{BxPC}-3$ gastrin knockdown clone were significantly smaller than any of the CCK shRNA clones or non-specific control tumors. Significantly different from NSC ${ }^{*} \mathrm{p}<0.05$.

and the corresponding gastrin and CCK mRNA levels in these tumors is shown in Fig. 6. BxPC-3 cells with normal gastrin and CCK mRNA levels formed large tumors in vivo (Fig. 6). Conversely, when gastrin expression was decreased without altering CCK expression, tumors were significantly smaller. These results indicate that pancreatic tumor growth is highly dependent on autocrine gastrin expression and that the amount of endogenous CCK produced by $\mathrm{BxPC}-3$ cells is insufficient to maintain growth in the absence of gastrin.

\section{Discussion}

The current investigation is the first to report the quantification of endogenous CCK mRNA and peptide in human pancreatic cancers and demonstrate the effect of downregulating $\mathrm{CCK}$ on tumor growth. Although conflicting publications both support (17) and oppose (16) the presence of endogenous CCK in human pancreatic cancer by immunohistochemistry, we demonstrate and confirm both the presence and quantity of CCK mRNA and peptide in pancreatic cancer by sensitive cellular and molecular techniques. Autocrine gastrin and its receptors have been known to play a role in proliferation of pancreatic cancer $(3,23)$ but the role of CCK has not been directly examined. In the current study, we demonstrate that human pancreatic cancer cells produce endogenous CCK; however, down-regulation of this peptide does not affect tumor cell growth in vivo. In pancreatic cancer cell lines that produce both peptides, gastrin is the predominant autocrine peptide influencing cell growth.

The reasons why only autocrine gastrin and not CCK seem to be important for pancreatic cancer growth may be related to the amount of these peptides produced. In this study, the amount of CCK peptide secreted by BxPC-3 cancer cells and measured by RIA is considerably less (228-fold) than that previously reported for gastrin (7). Perhaps the lower levels of CCK peptide may account for the inability to detect CCK immunoreactivity in pancreatic cancer specimens

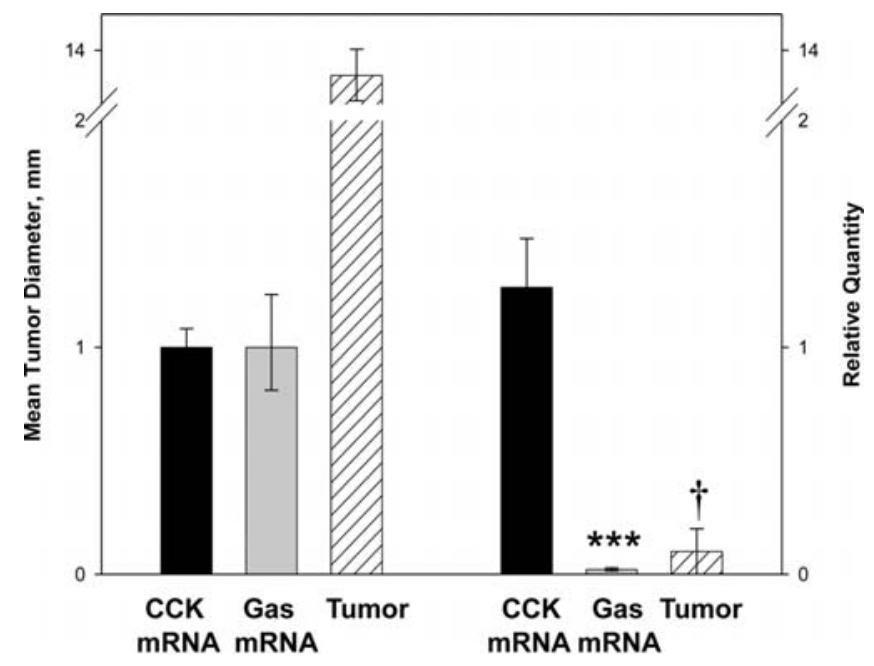

BxPC-3 Wild-type BxPC-3 Gastrin shRNA

Figure 6. In vivo pancreatic tumor growth in mice is dependent on autocrine gastrin but not autocrine CCK production. Subcutaneous tumors of wild-type BxPC-3 cells which express both gastrin and CCK reached a mean tumor diameter of $13.3 \mathrm{~mm}$ by week four. In BxPC-3 cells where only gastrin mRNA was down-regulated and CCK mRNA was unaltered (right), tumors were significantly smaller than wild-type tumors. Tumor size $(\mathrm{mm})$ is shown in the hatched columns. Significantly different from wild-type cells ${ }^{* * *} \mathrm{p}<0.0001$ and ${ }^{\dagger} \mathrm{p}<0.00001$.

examined by Goetze and colleagues (16). In view of the fact that most of the pancreatic cancer cell lines examined thus far secrete both gastrin (7) and CCK, it would be difficult to evaluate whether CCK itself contributes at all to cell proliferation since in the natural state gastrin appears to be the more abundant and ubiquitous peptide. Furthermore, since decreasing CCK does not alter tumor size, it is likely that growth is stimulated by the continued proliferative action of gastrin on the $\mathrm{CCK}_{2}$ receptor. In contrast, when gastrin is down-regulated in BxPC-3 cells without altering CCK levels, in vivo tumor growth is drastically reduced. These results indicate that although endogenous CCK is produced by tumor cells, this peptide is not adequate or sufficient to stimulate tumor growth by itself. Our study clearly shows that although the majority of human ductal pancreatic cancers produce both peptides, gastrin is the dominant peptide responsible for growth stimulation and tumor formation in vivo.

In this investigation, we also demonstrated that when the cells are exposed to exogenous peptides, equimolar concentrations of gastrin are more potent in stimulating growth of BxPC-3 pancreatic cancer cells than CCK. Previously we had confirmed that the predominant CCK receptor responsible for the proliferative actions of these peptides in pancreatic cancer was the $\mathrm{CCK}_{2}$ receptor $(4,7)$ or a spliced variant form of this receptor which retains the forth intron (6). When competition receptor binding assays were performed with either ${ }^{125} \mathrm{I}-\mathrm{CCK}$ (22) or $\left[\mathrm{H}^{3}\right]-\mathrm{L} 365,260$ (a $\mathrm{CCK}_{2}$ receptor antagonist), the affinity for gastrin at the $\mathrm{CCK}_{2}$ receptor was 50 -fold greater than that for CCK $(4,22)$. This increased affinity for gastrin over CCK to the $\mathrm{CCK}_{2}$ receptor in malignant tissues is unlike that reported for gastrin's at the $\mathrm{CCK}_{2}$ receptor in normal tissues where CCK and gastrin possess equal binding affinity 
$\left(\mathrm{K}_{\mathrm{d}}\right)(24)$ and may contribute to the greater effect demonstrated with gastrin.

The use of RNA interference (RNAi) technologies to reduce the expression of disease-related genes is becoming more widespread. Thus, a clear understanding of which gene products are modified by RNAi regulation and which nucleotide residues within a particular mRNA are the best targets for down-regulating gene expression is essential when developing new therapeutic agents. Because of their relatively small size, the mRNAs encoding peptides such as CCK and gastrin are especially amenable to regulation by RNAi technologies. Since these peptides act as growth factors for diseases such as pancreatic cancer, where few effective treatments are available clinically, development and testing of shRNAs targeting small peptides for cancer treatment is a critical area of research. In this study, we have defined two RNAi target sites within the human CCK mRNA that can effectively reduce the levels of this mRNA. Although these target sites will not be clinically relevant in the future treatment of pancreatic cancer, they may be useful in downregulation of CCK in other tissues.

The effectiveness of the down-regulation of target site may also be dependent on nucleotide position and secondary mRNA structure as well as post-transcriptional and translational events. Little clonal variation was observed with our shRNA transfections as compared to antisense techniques. Furthermore, shRNA location differences were found in that the shRNA +141 target site was more efficient in decreasing CCK mRNA levels than was the CCK shRNA -6 target site suggesting RNAi targeted close to the translation start site is less effective (25). Others have shown that the secondary structure of the mRNA target is the most critical factor in RNAi design (26). However, the results from the RIA in this report indicated post-transcriptional modifications affect the quantity of peptide produced which may be more important than the amount of mRNA available. Therefore, although the shRNA +141 clones had less mRNA, the peptide production and tumor growth was decreased more with the shRNA - 6 target site.

Another possible explanation for why significant CCK peptide down-regulation did not alter growth in pancreatic cancer may be that CCK mRNA is very stable or preferentially translated in order to maintain CCK peptides levels. This idea is unlikely since it is in conflict with other reports of concurrent mRNA and protein knockdown using a similar plasmid-based shRNA system with BxPC-3 cells. Wei et al recently reported that an shRNA directed against the Mcl-1 mRNA reduced both Mcl-1 mRNA and protein levels by $60-70 \%$ in BxPC-3, PANC-1 and SW1990 (27). The Mcl-1 shRNA expressing cells also formed smaller tumors than untransfected cells or vector-only and non-specific shRNA controls.

The current report confirms the expression of CCK peptide or mRNA in pancreatic cancer (17) and is the first report documenting the use of RNAi technology to downregulate $\mathrm{CCK}$ in this cancer. Our study validates two novel RNAi targets sites within the CCK mRNA: the mRNA sequences beginning at nucleotide -6 and at nucleotide +141 . Although a significant decrease in CCK mRNA was clearly achieved by these shRNA constructs, their utility in decreasing tumor growth in pancreatic cancer cells was not demonstrated most likely due to concomitant gastrin production. Using an shRNA to CCK mRNA in Ewing's tumors, Carrillo et al (28) were able to demonstrate $>90 \%$ down-regulation of CCK by real-time RT-PCR as well as decreased peptide secretion by RIA. Unfortunately, similar to our results, this group found that in spite of significant CCK suppression, tumor xenografts still grew well in nude mice suggesting growth factors other than CCK play an important role. We conclude that CCK can be efficiently down-regulated, but RNAi therapeutics that target gastrin would be a better strategy for pancreatic cancer treatment.

\section{Acknowledgements}

This research was supported by grants CA117926 and CA125445 from the National Cancer Institute to Dr J.P. Smith. Additional support was provided through an educational grant from the NIH, R25 DK078381. The technical assistance of Joseph Bednarczyk in the Pennsylvania State University College of Medicine Molecular Genetics Core Facility and Rob Brucklacher and Georgina Bixler in the Pennsylvania State University College of Medicine Functional Genomics Core Facility is greatly appreciated.

\section{References}

1. Pai SI, Lin YY, Macaes B, Meneshian A, Hung CF and Wu TC: Prospects of RNA interference therapy for cancer. Gene Ther 13: 464-477, 2006.

2. Smith JP, Kramer ST and Solomon TE: CCK stimulates growth of six human pancreatic cancer cell lines in serum-free medium. Regul Pept 32: 341-349, 1991.

3. Smith JP, Fantaskey AP, Liu G and Zagon IS: Identification of gastrin as a growth peptide in human pancreatic cancer. Am J Physiol 268: R135-R141, 1995.

4. Smith JP, Liu G, Soundararajan V, McLaughlin PJ and Zagon IS: Identification and characterization of CCK-B/gastrin receptors in human pancreatic cancer cell lines. Am J Physiol 266: R277-R283, 1994.

5. Smith JP and Zagon IS: Cholecystokinin receptors and human pancreatic adenocarcinomas. Int J Pancreatol 16: 243-246, 1994.

6. Smith JP, Verderame MF, McLaughlin P, Martenis M, Ballard E and Zagon IS: Characterization of the CCK-C (cancer) receptor in human pancreatic cancer. Int J Mol Med 10: 689-694, 2002.

7. Smith JP, Shih A, Wu Y, McLaughlin PJ and Zagon IS: Gastrin regulates growth of human pancreatic cancer in a tonic and autocrine fashion. Am J Physiol 270: R1078-R1084, 1996.

8. Bardram L, Hilsted L and Rehfeld JF: Progastrin expression in mammalian pancreas. Proc Natl Acad Sci USA 87: 298-302, 1990.

9. Smith JP, Hamory MW, Verderame MF and Zagon IS: Quantitative analysis of gastrin mRNA and peptide in normal and cancerous human pancreas. Int J Mol Med 2: 309-315, 1998.

10. Brand SJ and Fuller PJ: Differential gastrin gene expression in rat gastrointestinal tract and pancreas during neonatal development. J Biol Chem 263: 5341-5347, 1988.

11. Seretis EC, Gavriil AN, Golematis VC and VoloudakisBaltatzis IE: Immunoelectron study of pancreatic carcinomas using antibodies to gastrointestinal hormones. Ultrastruct Pathol 31: 303-314, 2007.

12. Grabowska AM and Watson SA: Role of gastrin peptides in carcinogenesis. Cancer Lett 257: 1-15, 2007.

13. Harris JC, Gilliam AD, McKenzie AJ, Evans SA, Grabowska AM, Clarke PA, McWilliams DF and Watson SA: The biological and therapeutic importance of gastrin gene expression in pancreatic adenocarcinomas. Cancer Res 64: 5624-5631, 2004.

14. Matters GL, Harms JF, McGovern CO, Jayakumar C, Crepin K, Smith ZP, Nelson MC, Stock H, Fenn CW, Kaiser J, Kester M and Smith JP: Growth of human pancreatic cancer is inhibited by down-regulation of gastrin gene expression. Pancreas 38 : e151-e161, 2009. 
15. Smith JP, Solomon TE, Bagheri S and Kramer S: Cholecystokinin stimulates growth of human pancreatic adenocarcinoma SW1990. Dig Dis Sci 35: 1377-1384, 1990.

16. Goetze JP, Nielsen FC, Burcharth F and Rehfeld JF: Closing the gastrin loop in pancreatic carcinoma: coexpression of gastrin and its receptor in solid human pancreatic adenocarcinoma. Cancer 88: 2487-2494, 2000.

17. Tamiolakis D, Venizelos I, Simopoulos C, Lambropoulou M, Kotini A, Jivannakis T, Alexiadis G, Boglou P and Papadopoulos N: Does neoplastic cholecystokinin expression reflect the embryonal pattern of the protein? A study in human pancreas. Acta Medica (Hradec Kralove) 47: 101-105, 2004.

18. Paloheimo LI and Rehfeld JF: A processing-independent assay for human procholecystokinin and its products. Clin Chim Acta 229: 49-65, 1994.

19. Livak KJ and Schmittgen TD: Analysis of relative gene expression data using real-time quantitative PCR and the 2(-Delta Delta C(T)) method. Methods 25: 402-408, 2001.

20. Weinberg DS, Ruggeri B, Barber MT, Biswas S, Miknyocki S and Waldman SA: Cholecystokinin A and B receptors are differentially expressed in normal pancreas and pancreatic adenocarcinoma. J Clin Invest 100: 597-603, 1997.

21. Gardner JD, Walker MD, Martinez J, Priestly GP, Natarajan S and Bodanszky M: The importance of the amino acid in position 27 of cholecystokinin in determining its biological activity on pancreatic acini. Biochim Biophys Acta 630: 323-329, 1980 .
22. Smith JP, Rickabaugh CA, McLaughlin PJ and Zagon IS: Cholecystokinin receptors and PANC-1 human pancreatic cancer cells. Am J Physiol 265: G149-G155, 1993.

23. Watson SA, Clarke PA, Smith AM, Varro A, Michaeli D, Grimes S, Caplin M and Hardcastle JD: Expression of CCKB/ gastrin receptor isoforms in gastro-intestinal tumour cells. Int $\mathbf{J}$ Cancer 77: 572-577, 1998

24. Amer MS: Studies with cholecystokinin. II. Cholecystokinetic potency of porcine gastrins I and II and related peptides in three systems. Endocrinology 84: 1277-1281, 1969.

25. Duxbury MS, Ito H, Zinner MJ, Ashley SW and Whang EE: EphA2: a determinant of malignant cellular behavior and a potential therapeutic target in pancreatic adenocarcinoma. Oncogene 23: 1448-1456. 2004.

26. Schubert KM, Scheid MP and Duronio V: Ceramide inhibits protein kinase $\mathrm{B} / \mathrm{Akt}$ by promoting dephosphorylation of serine 473. J Biol Chem 275: 13330-13335, 2000.

27. Wei SH, Dong K, Lin F, Wang X, Li B, Shen JJ, Zhang Q, Wang $\mathrm{R}$ and Zhang HZ: Inducing apoptosis and enhancing chemosensitivity to gemcitabine via RNA interference targeting Mcl-1 gene in pancreatic carcinoma cell. Cancer Chemother Pharmacol 62: 1055-1064, 2008.

28. Carrillo J, Garcia-Aragoncillo E, Azorin D, Agra N, Sastre A, Gonzalez-Mediero I, Garcia-Miguel P, Pestana A, Gallego S, Segura D and Alonso J: Cholecystokinin down-regulation by RNA interference impairs Ewing tumor growth. Clin Cancer Res 13: 2429-2440, 2007. 